\title{
Prediction of Fractional-Order Chaotic Systems Behavior Using NARX Neural Networks
}

\author{
Reza Behinfaraz*, Sehraneh Ghaemi, Sohrab Khanmohammadi \\ Faculty of Electrical and Computer Engineering University of Tabriz, Tabriz, Iran. \\ * Corresponding author. Tel:: 0098-413-3393740; email: r.behinfaraz@tabrizu.ac.ir \\ Manuscript submitted August 10, 2017; accepted September 18, 2017. \\ doi: 10.17706/ijcee.2018.10.1.23-30
}

\begin{abstract}
Chaotic systems are complex systems which have very different behavior in different situation. When a chaotic system is fractional-order type, this complexity become more than past. Then prediction of these systems behavior is an important task which has many application in many research field. In this paper we propose a method based on artificial neural networks to prediction of this systems behavior in different conditions. Finally simulation examples show the performance of the proposed method.
\end{abstract}

Key words: Fractional-order chaotic system, NARX neural network, prediction.

\section{Introduction}

Chaotic systems and fractional calculation have an old history in many research fields. According to the wide application of fractional-order chaotic systems, analysis of these systems behavior becomes one of the important tasks in this area. Chaotic behavior of fractional-order systems was shown in many papers in this field [1]-[4]. In fractional order chaotic systems, in addition to the parameters of system, orders of systems play important role in chaotic behavior of these systems and fractional orders make these systems more complex than integer version of these systems.

Many process are described by time series nowadays. It means that these systems have dependence to previous sequence of systems and in general form it can be shown with

$$
y(t), t=0,1,2, \ldots
$$

The application of prediction or forecasting of time series is largely presented in many studies [5]-[7].

Prediction of chaotic systems behavior is a kind of observer-based synchronization, which has many applications such as in secure communications [18].

One of the most important properties of fractional-order systems is dependence of these systems to past samples of systems. In other word, fractional systems are systems with memory. According to this point and structure of NARX neural networks, it seems that this networks must be an useful tools to approximate of fractional-order systems behavior, then in this paper we use this networks to predict the behavior of fractional-order chaotic systems.

This paper is organized as the follows: In Section 2, we introduce some basic properties and relations about fractional order systems. Also we describe the structure of NARX neural network structure in this section. In Section 3, we propose two well-known fractional-order systems and also parameters of NARX 
system structure are defined in this section. In Section 4, we simulate the proposed method to prediction of states of systems in Section 3. Finally in Section 5 we conclude this paper and show the effectiveness of the proposed method to this problem.

\section{Problem Definition}

\subsection{Neural Network for Prediction}

Neural network tools to prediction of time series are nonparametric and it is not necessary to know the specification of system. Recurrent Neural Networks (RNN) with sufficient number of neurons can be a realization of the nonlinear systems. [8].

According to the Neural Networks(NN) approximation properties, NN based prediction has been developed more than past. Many researchers are worked on these issues using neural networks, for modeling and prediction. However, the proposed methods still don't have full performance and many limitations remain in their, then many researcher work on new neural networks models and learning techniques [8]-[12]. Wide range of data for training neural networks, make these systems with difficulties in modeling and it causes low accuracy in the response of systems. Also the researches show that the large number of training data dose not always help to improve the accuracy of prediction.

Prediction of time series is a kind of system identification techniques. The main purpose of this paper is creating system model which can approximate the nonlinear system which produce the complex time series. The model system is considered as an artificial RNN. The structure of RNN is contained a weighted feedback connection between layers of neurons also it can add more than one time as feedback delays. Therefore, it seems use of this architecture is a suitable choice for prediction of time series.

\subsection{Structure}

The base architecture of RNN for the proposed method to predict chaotic behavior of fractional-order systems is considered as a Nonlinear Autoregressive with eXogeneous inputs (NARX) neural networks, in this paper [10].

It was shown that this type of neural networks is a powerful type of neural networks for modeling of nonlinear systems. Also in comparison with to the other types of neural networks, learning in NARX structure is more effective and in general, this type of learning algorithm converges faster than other types[10], [13].

In this structure each outputs of system at the discrete time instant $t$ related to past outputs and other inputs with

$$
y(t)=f\left(y(t-1), \ldots, y\left(t-n_{y}\right), x(t-1), \ldots . ., x\left(t-n_{x}\right)\right)
$$

where $y$ and $x$ are the inputs of the model and $y(t)$ is the output of model at a discrete time step $t$. Also in this model, $n_{y} \geq 1, n_{x} \geq 1$ are the number of input memory and output memory delays and $f$ is a nonlinear function, and this function is approximated by a multilayer perceptron (MLP). Overall , a NARX network consists of a MLP network inside it, and this MLP network has relation with independent inputs and past outputs to calculate output. In this type of network as shown in Fig.(1), the feedback of system is defined using the system output and also independent input and there is not any feedback from outputs of hidden layer of network, nevertheless output of NARX networks more accurate than other types of recurrent neural networks [10]. Basic structure of this model is shown in Fig. (1). 


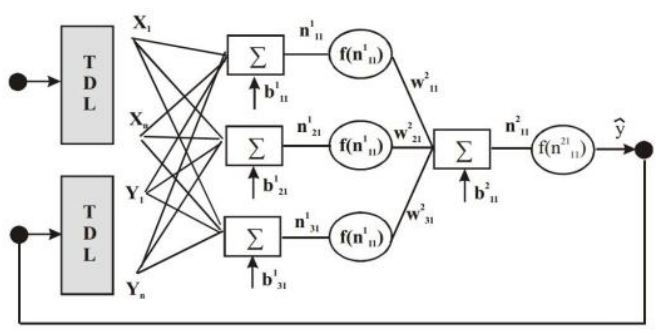

Fig. 1. Structure of NARX model.

\subsection{Fractional Calculations}

\subsubsection{Definition of fractional operator}

Describing of a fractional-order system needs to solve fractional-order differential equations. There are three more common definition of fractional-order operator: Grunwald-Letnikov" (GL) definition, Riemann-Liouville(RL) definition and Caputo definition [14].

According to the discrete version of GL definition, we use this definition in this paper. The GL definition of fractional operator is:

$$
{ }_{a} D_{t}^{\alpha} f(t)=\lim _{h \rightarrow 0} h^{-\alpha} \sum_{j=0}^{\left[\frac{t-a}{h}\right]}(-1)^{j}\left(\begin{array}{c}
\alpha \\
j
\end{array}\right) f(t-j h)
$$

where

$$
\left(\begin{array}{l}
\alpha \\
j
\end{array}\right)=\frac{\alpha !}{j !(n-j) !}
$$

\subsubsection{Stability theorem of fractional-order system}

In this subsection we study on stability conditions and regions of fractional-order system . For the following fractional differential equation:

$$
D^{\alpha} x=A x, \quad x(0)=x_{0}
$$

where $x \in R^{n}, A \in R^{n \times n}$ and

$$
\alpha=\left[\alpha_{1}, \alpha_{2}, \ldots, \alpha_{n}\right], \quad\left(0<\alpha_{i} \leq 1\right)
$$

for $(i=1,2, \ldots, n)$ shows the fractional orders. For $\alpha_{1}=\alpha_{2}=\ldots=\alpha_{n}=\alpha$, the fractional-order system (4) is asymptotically stable if

$$
|\arg (\operatorname{spec}(A))|>\alpha \pi / 2
$$

In this condition the states converges to 0 . For $\alpha=1$, the above condition is changed to the ordinary linear differential systems stability condition.

Suppose that $\alpha_{i}$ s are not equal and also these numbers are rational numbers between 0 and 1 . Also we suppose

$$
\alpha_{i}=\frac{v_{i}}{u_{i}}, \operatorname{rem}\left(u_{i}, v_{i}\right)=1
$$

where $u_{i}, v_{i} \in N$, for $i=1,2, \ldots, n$. If all the roots of the equation

$$
\operatorname{det}(\operatorname{diag}(M 1, M 2, \ldots, M n) A)=0
$$


$M$ is the least common multiple of the dominator $u_{i}$ of $\alpha_{i}^{\prime} s$, satisfy

$$
|\arg (\lambda)|>\pi / 2 M
$$

Then system (4) is asymptotically stable.

In the follows, we can use the above stability theorem to ensure that fractional-order system is not asymptomatically stable and this is one of necessary conditions to chaotic behavior of a fractional-order system.

\subsubsection{Numerical algorithm for fractional-order systems}

According to the non-analytically solutions of fractional order differential equation, we must use an approximation method to solve this type of differential equations. There are some method to solve fractional-order differential equations. In this paper we use Adams-Bashforth-Moulton method to solve this type equations. To describe this method, consider the following differential equation:

$$
D^{\alpha} y(t)=r(t, y(t)), 0<t \leq T
$$

And

$$
y^{\left(k^{\prime}\right)}(0)=y_{0}^{k^{\prime}}, k^{\prime}=0,1, \ldots, m-1
$$

This differential equation is equivalent to Volterra integral equation by [14]:

$$
\mathrm{y}(\mathrm{t})=\sum_{\mathrm{k}^{\prime}=0}^{\alpha-1} \mathrm{y}_{0}^{\left(\mathrm{k}^{\prime}\right)} \frac{\mathrm{t}^{\mathrm{k}^{\prime}}}{\mathrm{k}^{\prime} !}+\frac{1}{\Gamma(\alpha)} \sum_{j=0}^{k} b_{j, k+1} r\left(t_{j}, y_{h}\left(t_{j}\right)\right)
$$

where $\lceil\alpha\rceil$ shows first integer number bigger than $\alpha$.

Now, with $h=T / N, t_{k}=k h(n=0,1,2, \ldots, N)$

Then Eq. (5) can be discretized as follows:

$$
\mathrm{y}_{\mathrm{h}}\left(\mathrm{t}_{\mathrm{k}+1}\right)=\sum_{\mathrm{k}^{\prime}=0}^{\alpha-1} \mathrm{y}_{0}^{\left(\mathrm{k}^{\prime}\right)} \frac{\mathrm{t}_{\mathrm{k}+1}^{\mathrm{k}^{\prime}}}{\mathrm{k}^{\prime} !}+\frac{\mathrm{h}^{\alpha}}{\Gamma(\alpha+2)} \mathrm{r}\left(\mathrm{t}_{\mathrm{n}+1}, \mathrm{y}_{\mathrm{h}}^{\mathrm{P}}\left(\mathrm{t}_{\mathrm{n}+1}\right)\right)+\frac{\mathrm{h}^{\alpha}}{\Gamma(\alpha+2)} \sum_{\mathrm{j}=0}^{\mathrm{n}} \mathrm{a}_{\mathrm{j}, \mathrm{n}+1} \mathrm{r}\left(\mathrm{t}_{\mathrm{j}}, \mathrm{y}_{\mathrm{h}}^{\mathrm{P}}\left(\mathrm{t}_{\mathrm{j}}\right)\right)
$$

where predicted value $y_{h}\left(t_{n+1}\right)$ is determined by

$$
\mathrm{y}(\mathrm{t})=\sum_{\mathrm{k}^{\prime}=0}^{\alpha-1} \mathrm{y}_{0}^{\left(\mathrm{k}^{\prime}\right)} \frac{\mathrm{t}^{\mathrm{k}^{\prime}}}{\mathrm{k}^{\prime} !}+\frac{1}{\Gamma(\alpha)} \sum_{j=0}^{k} b_{j, k+1} r\left(t_{j}, y_{h}\left(t_{j}\right)\right)
$$

in which

$$
\mathrm{a}_{\mathrm{j}, \mathrm{k}+1}=\left\{\begin{array}{c}
\mathrm{k}^{\alpha+1}-(\mathrm{k}-\alpha)(\mathrm{k}+1)^{\alpha}, j=0 \\
(\mathrm{k}-\mathrm{j}+2)^{\alpha+1}+(\mathrm{k}-\mathrm{j})^{\alpha+1}-2(\mathrm{k}-\mathrm{j}+1)^{\alpha+1}, 1 \leq j \leq N \\
1, j=k+1 \\
\mathrm{~b}_{\mathrm{j}, \mathrm{k}+1}=\frac{\mathrm{h}^{\alpha}}{\alpha}\left((\mathrm{k}+1-\mathrm{j})^{\alpha}(\mathrm{k}-\mathrm{j})^{\alpha}\right)
\end{array}\right.
$$

The estimation error of this approximation is described as follows:

$$
\max \left|y\left(t_{j}\right)-y_{h}\left(t_{j}\right)\right|=o\left(h^{P}\right)
$$


where $p=\min (2,1+\alpha)$.

\section{System Description}

\subsection{Fractional Duffing System}

Duffing system has been applied in many fields, for example, fluid flow induced vibration, large amplitude oscillation of centrifugal governor systems and mathematical modeling and so on[15]-[17].

The Duffing system integer differential equation is described by

$$
m \frac{d^{2} x}{d t^{2}}+c \frac{d x}{d t}+k x(t)+a x^{3}(t)=f \sin (w t)
$$

In this differential equation, $m$ is the mass, $c$ is the damping factor, $k$ is the coefficient of linear rigidity, $a$ is the coefficient of nonlinear stiffness, $f$ is the amplitude of the excitation and $\omega$ is the frequency of the excitation.

Fractional version of Duffing system in state space representation can be shown as[13].

$$
\left\{\begin{array}{c}
D^{\alpha_{1}} x=y \\
D^{\alpha_{2}} y=\frac{1}{m}\left(f \sin (w t)-a x^{3}-k x-c y\right.
\end{array}\right.
$$

where $\alpha_{1}$ and $\alpha_{2}$ are the fractional-order of system. The chaotic attractor of this systems with $\alpha_{1}=0.9, \alpha_{2}=1$ and selected parameters as $c=-0 . .15, f=0.3, m=1, k=-1, a=1, \omega=1$ is shown in Fig. (2).

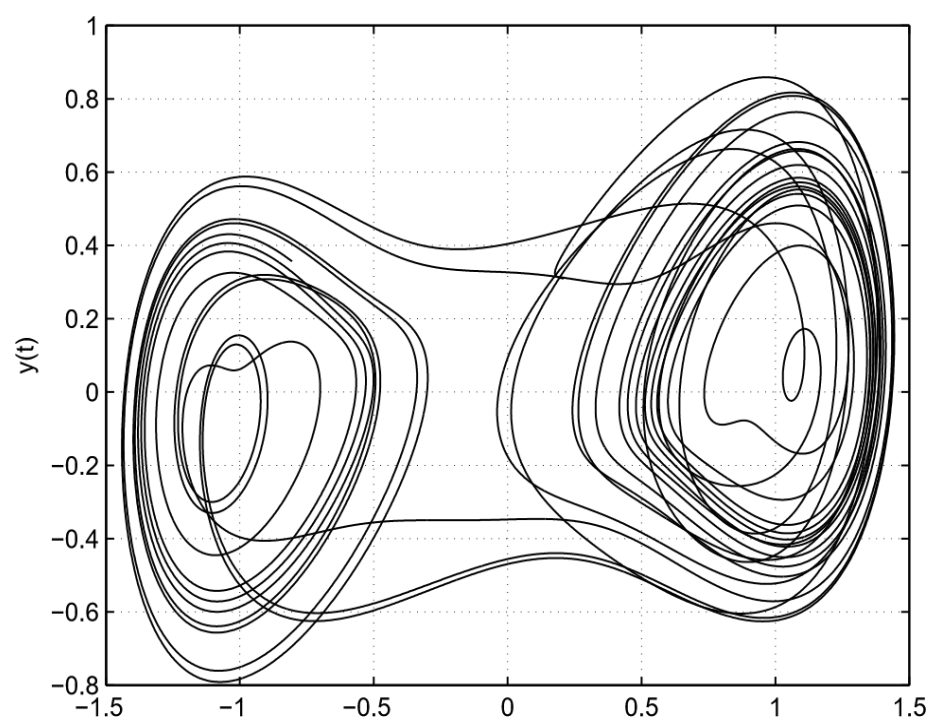

Fig. 2. Chaotic behavior of fractional-order duffing system.

\section{Simulations}

In this section we simulate the fractional-order Duffing system with mentioned orders and parameters in previous section. To predict the states of this system we use a NARX network with one hidden layer. Size of this layer is considered 10. In the each state, other states is considered as an independent input in relation (2). Training data of NARX networks for two states is selected by discretized samples form 0 to 12 seconds. Used discretization method was described in section 2. Also Time step in discretization is considered 0.005 seconds. For increasing the performance of the proposed method, number of delayed feedback which has 
affect in each state output is selected 100 samples by trial and error. It means that each state has dependence to 100 previous samples $\mathrm{f}$ that and other states and this is the one of important point of the proposed method to get convergence as fast as possible. As shown in Fig. (3 \& 4), after training networks in first 12 seconds with above parameters, the convergence of prediction of system states are occurred in acceptable times. Also the errors in this prediction are illustrated in Fig. (5 \& 6).

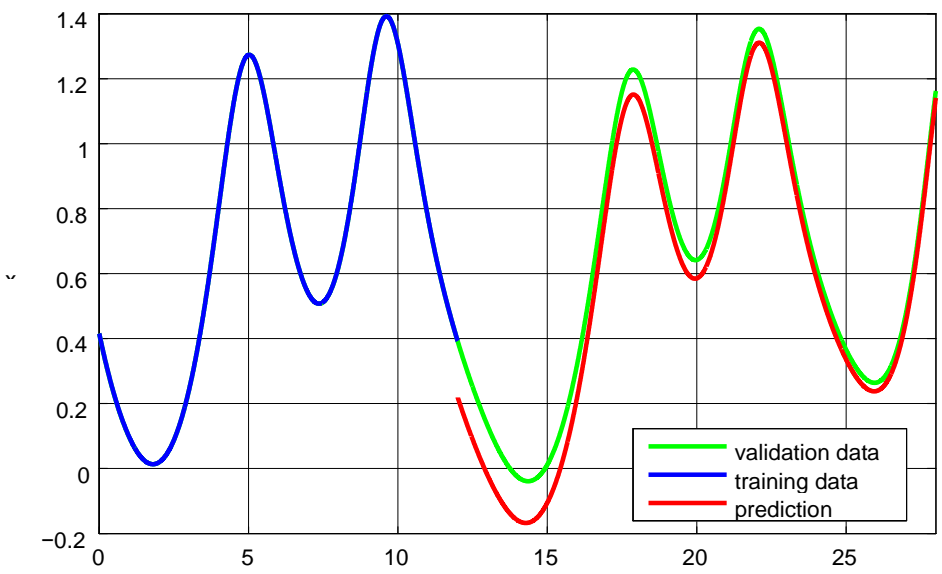

Fig. 3. First state of duffing system training, validation and prediction.

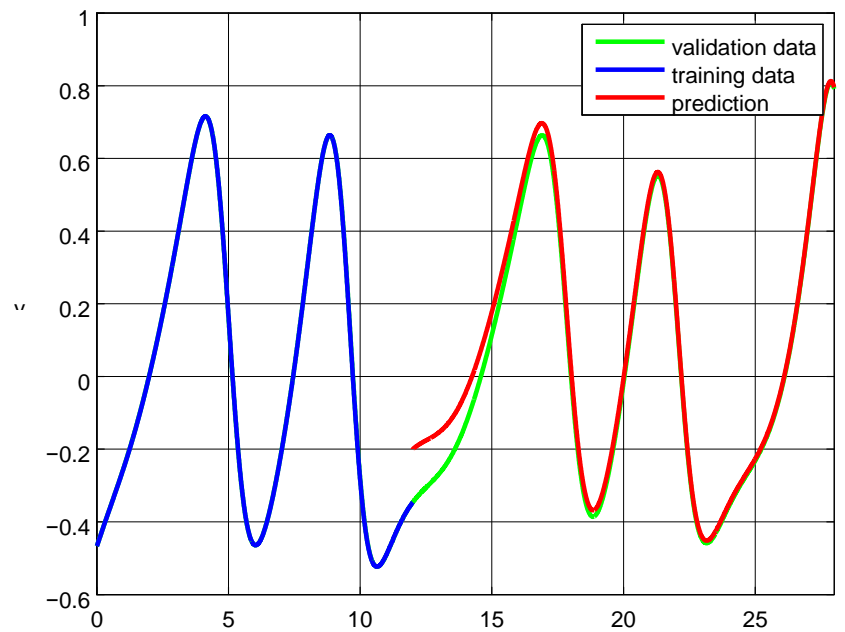

Fig. 4. Second state of duffing system training, validation and prediction.

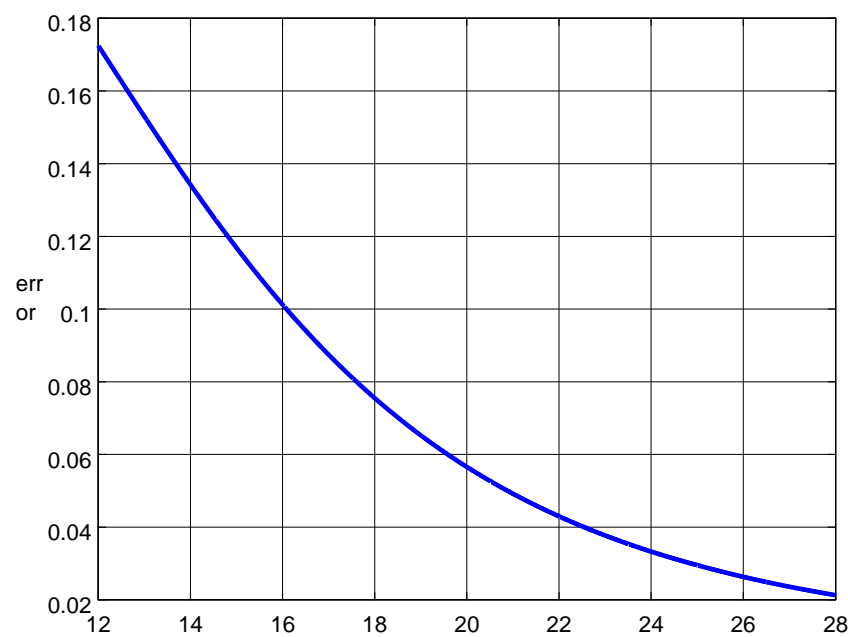

Fig. 5. Prediction error of first state of duffing system using NARX network. 


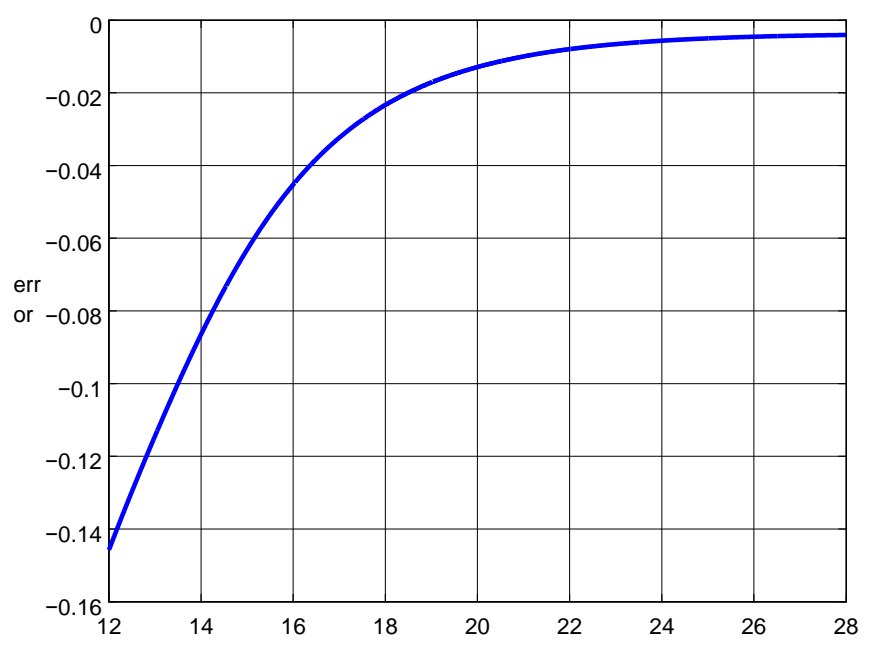

Fig. 6. Prediction error of second state of Duffing system using NARX network.

\section{Conclusion}

In this paper prediction of chaotic behavior of fractional order chaotic system is discussed. The proposed method is used for fractional-order Duffing system. The prediction method is defined using NARX neural networks. An example is simulated to show the performance of the proposed method. Although fractional-order chaotic systems have more complexity against ordinary chaotic systems but results show that by selecting a good parameters for NARX networks we can get accurate prediction of chaotic behavior of fractional order chaotic systems.

\section{References}

[1] Hartley, T. T., Lorenzo, C. F. \& Killory Qammer, H. (1995). Chaos in a fractional order Chua's system. IEEE Transactions on Circuits and Systems I: Fundamental Theory and Applications, 42(8), 485-490.

[2] Behinfaraz, R., \& Badamchizadeh, M. A. (2015). Synchronization of different fractional-ordered chaotic systems using optimized active control. Proceedings of 2015 6th International Conference on Modeling, Simulation, and Applied Optimization (ICMSAO) (pp. 1-6).

[3] Behinfaraz, R., \& Badamchizadeh, M. A. (2015). New approach to synchronization of two different fractional-order chaotic systems. Proceedings of 2015 International Symposium on Artificial Intelligence and Signal Processing (AISP) (pp. 149-153).

[4] Reza, B., \& Mohammadali, B. (2016). Optimal synchronization of two different in-commensurate fractional-order chaotic systems with fractional cost function.

[5] Tertisco, M., Stoica, P., \& Petrescu, T. (1985). Modeling and forecasting of time series. Publ House of Romanian Academy.

[6] Pollok, D. S. G. (2001). A handbook of time series, signal processing and dynamics. Academic Press.

[7] Edgar, E. P. (2001). Fractal market analysis. John Wiley \& Sons.

[8] Gao, Y., \& Meng, J. E. (2005). NARMAX time series model prediction: Feedforward and recurrent fuzzy neural network approaches. Fuzzy Sets and Systems, 150(2), 331-350.

[9] Hagan, M. T., Jesus, O. D., \& Schultz, R. (2001). Training recurrent networks for filtering and control. In L. R. Medsker, \& L. C. Jain (Eds.), Recurrent Neural Networks Design and Applications. CRC Press.

[10] Tsungnan, L., Lee Giles, C., Horne, B. G., \& Kung, S. Y. (1997). A delay damage model selection algorithm for NARX neural networks. IEEE Transactions on Signal Processing, Special Issue on Neural Networks, 45(11), 2719-2730.

[11] Siegelmann, H. T., Horne, B. G., \& Lee Giles, C. Computational capabilities of recurrent NARX neural 
networks. IEEE Transactions on Systems, Man and Cybernetics, Part B, 27.

[12] Tsungnan, L., Horne, B. G., Tino, P., Lee Giles, C. (1996). Learning long-term dependencies in NARX recurrent neural networks. IEEE Transactions on Neural Networks, 7(6), 1329-1351.

[13] Li, Z. S., Chen, D. Y., Zhu, J. W., \& Liu, Y. J. (December 2015). Nonlinear dynamics of fractional order Duffing system. Chaos, Solitons \& Fractals, 81(A), 111-116.

[14] Reza, B., Mohammadali, B., \& Amir Rikhtegar, G. (April 2016). An adaptive method to parameter identification and synchronization of fractional-order chaotic systems with parameter uncertainty. Applied Mathematical Modelling, 40(7-8), 44684479.

[15] Narakorn, S., \& Hossein, Z. (October 2012). Modelling of coupled crossflow/in-line vortex-induced vibrations using double Duffing and van der Pol oscillators. Ocean Engineering, 53, 83-97.

[16] Jimenez, S., Gonzalez, J. A., Vazquez, L. (2013). Fractional Duffings equation and geometrical resonance. Int J Bifurc Chaos, 23, 1350089.

[17] Brzeski, P., Perlikowski, P., \& Kapitaniak, T. (2014). Numerical optimization of tuned mass absorbers attached to strongly nonlinear duffing oscillator. Commun Nonlinear Sci Numer Simul, 19, 298-310.

[18] Zhu, F. L. (June 2009). Observer-based synchronization of uncertain chaotic system and its application to secure communications. Chaos, Solitons \& Fractals, 40(5), 2384-2391.

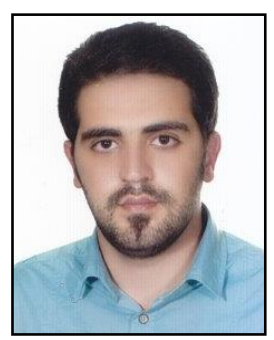

Reza Behinfaraz was born in Tabriz, Iran on May 14th, 1990. He received B.Sc. degree in electronic engineering from university of Tabriz and the M.Sc. degree in control engineering from University of Tabriz. He is currently working toward the Ph.D degree at university of Tabriz in control engineering. His fields of study and research was in: soft computing and intelligent systems, robotic, chaotic systems and fractional calculation.

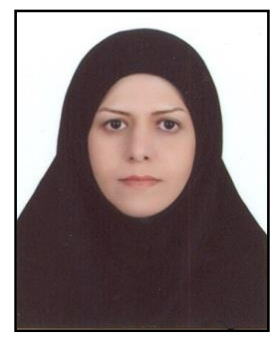

Sehraneh Ghaemi is currently an associate professor of Control Department with the Faculty of Electrical and Computer Engineering, University of Tabriz, Tabriz, Iran. She received her B.Sc. degree in power engineering from Amir Kabir University, Tehran, Iran in 1999, master and Ph.D in control engineering from University of Tabriz, Tabriz, Iran in 2003 and 2010. She is the author or a coauthor of more than 50 papers that have appeared in various journals and conference proceedings. Her main research interests are type 2 fuzzy systems, fuzzy systems, evolutionary algorithms, fuzzy-neural networks, intelligent control. Email: ghaemi@tabrizu.ac.ir

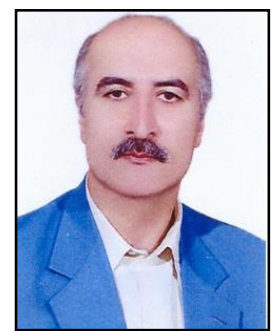

Sohrab Khanmohammadi is a professor of control engineering in Faculty of Electrical and Computer Engineering, University of Tabriz, Iran. He received his BSC in industrial engineering from Sharif University of Technology in 1981, Iran, MSC in automatic from Paul Sabatier University, DES and Ph.D in advanced automatic and system from National Institute of Aeronautics and Space in France. His research interests include fuzzy systems, computer simulation techniques, reliability, decision making and project control. 